\title{
Failing beta-cell adaptation in South Asian families with a high risk of type 2 diabetes
}

\author{
Sjaam Jainandunsing • Behiye Özcan • Trinet Rietveld • \\ Joram N. I. van Miert • Aaron J. Isaacs • Janneke G. Langendonk • \\ Felix W. M. de Rooij • Eric J. G. Sijbrands
}

Received: 1 February 2014/Accepted: 12 April 2014/Published online: 5 May 2014

(C) The Author(s) 2014. This article is published with open access at Springerlink.com

\begin{abstract}
We performed an extended oral glucose tolerance test (OGTT) to investigate the relationship between early and late beta-cell response and type 2 diabetes (T2D) in families of South Asian origin and indigenous Dutch, burdened by T2D. Based on the OGTT, 22 individuals were normoglycemic, 12 glucose intolerant and 23 had T2D in the South Asian families; these numbers were 34, 12 and 18 in the Caucasian families, respectively. The OGTT had 11 blood samplings in $3.5 \mathrm{~h}$ for glucose, insulin and C-peptide measurements. Through early and late insulin secretion rate (ISR), the above basal glucose areaunder-the-curve after glucose load (glucose disposal) and insulin sensitivity index (ISI), we obtained early and late disposition indices (DI). South Asians on average had lower ISI than Caucasians $(3.8 \pm 2.9$ vs. $6.5 \pm 4.7$, respectively, $P<0.001$ ), with rapid decline of their early and late DI between normal glucose tolerance versus impaired fasting glucose/impaired glucose tolerance (late DI; $P<0.0001)$. Adjusted for ISI, age, gender and waist-
\end{abstract}

Managed by Antonio Secchi.

Electronic supplementary material The online version of this article (doi:10.1007/s00592-014-0588-9) contains supplementary material, which is available to authorized users.

S. Jainandunsing $\cdot$ B. Özcan $\cdot$ T. Rietveld .

J. N. I. van Miert · J. G. Langendonk ·

F. W. M. de Rooij ( $₫)$. E. J. G. Sijbrands

Department of Internal Medicine, Erasmus MC - University

Medical Center Rotterdam, Room Bd-299, PO Box 2040,

3000 CA Rotterdam, The Netherlands

e-mail: f.derooij@erasmusmc.nl

A. J. Isaacs

Department of Epidemiology, Erasmus MC - University Medical

Center Rotterdam, Rotterdam, The Netherlands to-hip ratio, early ISR was significantly associated with glucose disposal in South Asians ( $\beta=0.55[0.186 ; 0.920])$, but not in Caucasians $(\beta=0.09[-0.257 ; 0.441])$. Similarly, early ISR was strongly associated with late ISR $\left(\beta=0.71[0.291 ; 1.123] ; R^{2}=45.5 \%\right)$ in South Asians, but not in Caucasians $(\beta=0.27[-0.035 ; 0.576]$; $R^{2}=17.4 \%$, with significant interaction between ethnicity and early ISR $(\beta=0.341[0.018 ; 0.664])$. Ordinal regression analyses confirmed that all South Asian OGTT subgroups were homogenously resistant to insulin and solely predicted by early ISR $(\beta=-0.782[-1.922$; 0.359], $\beta=-0.020[-0.037 ;-0.002]$, respectively), while in Caucasian families both ISI and early ISR were related to glucose tolerance state $(\beta=-0.603[-1.105 ;-0.101]$, $\beta=-0.066[-0.105 ;-0.027]$, respectively). In South Asian individuals, rapid beta-cell deterioration might occur under insulin resistant conditions. As their early insulin response correlates strongly with both glucose disposal and late insulin response, alterations in beta-cell dynamics may give an explanation to their extreme early onset of T2D, although larger prospective studies are required.

Keywords Type 2 diabetes - OGTT: insulin - Glucose . South Asian

$\begin{array}{ll}\text { Abbreviations } \\ \text { CIR } & \begin{array}{l}\text { Corrected insulin response } \\ \text { DI }\end{array} \\ \text { DOMA-B } & \begin{array}{l}\text { Homeostion index } \\ \text { function }\end{array} \\ \text { IGI } & \text { Insulinogenic index } \\ \text { ISEC } & \text { Insulin SECretion } \\ \text { ISI } & \text { Insulin sensitivity index } \\ \text { ISR } & \text { Insulin secretion rate } \\ \text { MRCi } & \text { Metabolic clearance rate of insulin }\end{array}$




\section{Introduction}

Dutch citizens of South Asian origin have a nearly fivefold higher prevalence of type 2 diabetes (T2D) than the indigenous Dutch population (further described as Caucasian) $[1,2]$. The increased susceptibility to T2D is also evident from the early onset of the disorder at relatively low body mass and the remarkably high incidence of cardiovascular and microvascular damage among the South Asians [2, 3]. A number of factors have been proposed to account for this strikingly high risk in South Asians, including a high prevalence of metabolic syndrome, impaired maternal lipid profile conditions, low birth weight causing central obesity later in life, dysfunction of adipocytes, as well as educational, social and economic inequalities [4-14]. These factors all enhance insulin resistance and promote hyperinsulinemia [14, 15]. In addition, T2D is characterized by beta-cell dysfunction. Genetic loci predisposing individuals to T2D affect both beta-cell function and insulin action [16, 17].

The ancestors of South Asian families in the Netherlands moved from a circumscribed region in India to Surinam. During the past 150 years, these South Asian families lived largely in genetic isolation before arriving in the Netherlands. The conservation of susceptibility loci may have contributed to the strong aggregation of T2D in these families. We hypothesized that, in addition to severe resistance to insulin, these South Asian families are also predisposed to develop beta-cell dysfunction. Therefore, we investigated beta-cell function and insulin sensitivity simultaneously in South Asian and Caucasian patients with T2D and first-degree relatives. In effect, we assessed the contribution to the risk of T2D of changes in early and late insulin secretion rates (ISR) and insulin sensitivity during an extended oral glucose tolerance test (OGTT) with insulin and C-peptide measurements.

\section{Methods}

\section{Subjects}

The study was conducted during a time period between August 2007 and January 2011. Patients with T2D and first-degree relatives without T2D were recruited from 36 South Asian families and 24 Caucasian families (Scheme 1). Power calculation was performed with Quanto version 1.0 [31] and was based on differences in early phase ISR (described further on in Methods section) between healthy South Asian and Caucasian performed in a pilot phase of the study among, with alpha 0.05 and power $80 \%$. All probands were attending the outpatient clinic of the Department of Internal Medicine of the Erasmus Medical Center in Rotterdam. T2D was diagnosed according to World Health Organization (WHO) criteria [18]: plasma glucose level $\geq 7.0 \mathrm{mmol} / \mathrm{L}$ in a fasting state and $/$ or $\geq 11.1 \mathrm{mmol} / \mathrm{L}$ in a non-fasting state. Inclusion criteria for probands were age of 18 years or older and T2D in at least one sibling. Both parents of the South Asian probands were of South Asian origin, and Caucasian probands were born in the Netherlands with both parents of Caucasian Dutch origin. Exclusion criteria were insulindependent diabetes mellitus, using medication other than metformin, a history of pancreatitis, insulinoma or other reasons that made participation impossible. Written informed consent was obtained from all participants. The study protocol was approved by the Erasmus Medical Center Medical Ethics Review Board.

Physical examination

Body height and weight were measured to the nearest $0.1 \mathrm{~cm}$ and $0.1 \mathrm{~kg}$ for the determination of body mass index (BMI). Waist circumference was measured in $\mathrm{cm}$ halfway between the lowest rib and the iliac crest, the maximum circumference of the hips was measured in the standing position in $\mathrm{cm}$, and, from these measurements, the waist-to-hip ratio $(\mathrm{W} / \mathrm{H})$ was calculated. Systolic and diastolic blood pressures were measured with an electronic blood pressure monitor (Datascope Accutorr Plus Inc., Montvale, NJ) after five minutes rest in the sitting position.

Oral glucose tolerance test (OGTT)

Glucose, $75 \mathrm{~g}$ dissolved in $200 \mathrm{ml} \mathrm{H}_{2} \mathrm{O}$, was administered orally after a 10-h overnight fast. Venous blood was drawn via an intravenous canula, 60 and $15 \mathrm{~min}$ before the glucose load and 15, 30, 45, 60, 90, 120, 150, 180 and $210 \mathrm{~min}$ after glucose loading. WHO criteria based on OGTT were used to define family members with normal glucose tolerance (NGT), impaired fasting glucose/impaired glucose tolerance (IFG/IGT) and T2D [18].

\section{Assays}

Plasma glucose was measured by a hexokinase-based method (Gluco-quant; Roche Diagnostics, Mannheim, Germany). Plasma insulin and C-peptide were measured separately by a competitive chemiluminescent immunoassay, supplied by Euro/DPC (Diagnostic Product Corporation, Los Angeles, CA). The assay was performed on a DPC Immulite 2000 analyzer (Euro/DPC) according to the manufacturer's recommended protocol. 


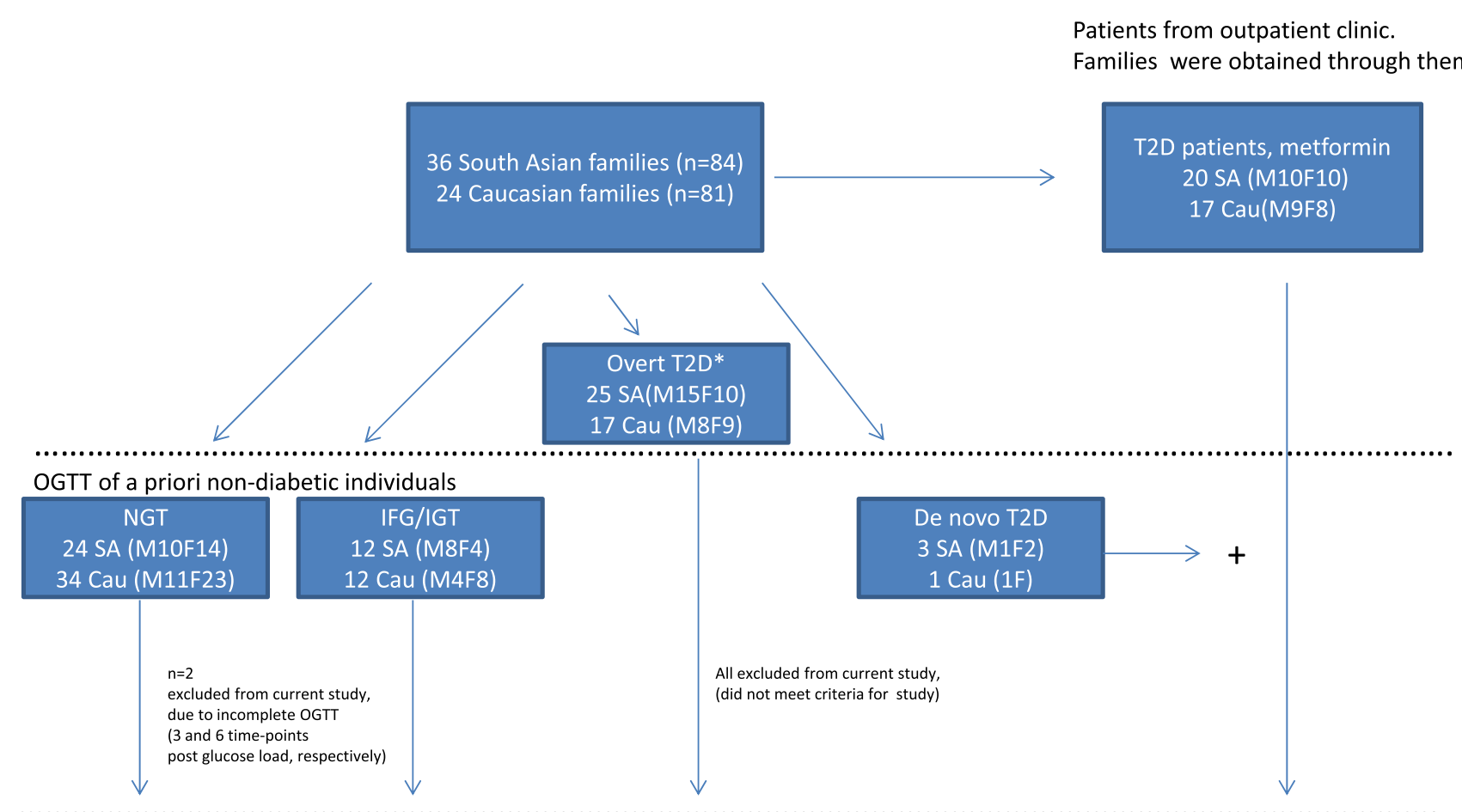

Used for current study

\begin{tabular}{c}
\hline NGT \\
22 SA (M10F12) \\
34 Cau (M11F23) \\
\hline
\end{tabular}
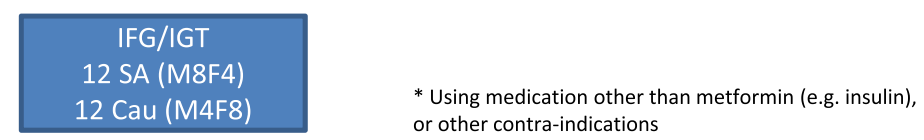

Scheme 1 Inclusion flow chart of individuals from South Asian and Caucasian families

\section{Calculation of indices}

\section{Beta-cell function indices}

For the assessment of early, late and overall beta-cell function, we calculated incremental ISR area-under-the curves (AUCs); ISR t0-30 and ISR t60-210 and ISR t0-210, respectively, based on plasma C-peptide concentrations with ISEC software [19]. The ISR reflects the prehepatic secretion rate, as $\mathrm{C}$-peptide has negligible hepatic clearance. Hereafter, we investigated early, late and overall beta-cell function in relation to glucose concentrations and insulin sensitivity to obtain early, late, and overall disposition indices (DI), respectively.

\section{Insulin sensitivity}

The insulin sensitivity index (ISI) was determined according to $[20,21]$

$$
\begin{aligned}
\mathrm{ISI}= & 10.000 /\left(G_{0} \times I_{0} \times \text { mean } G_{0-30-60-90-120}\right. \\
& \left.\times \text { mean } I_{0-30-60-90-120}\right)^{1 / 2} .
\end{aligned}
$$

\section{Early, late and overall Disposition Indices}

Early, late and overall DI were calculated as follows: ISR t0-30/glucose disposal t0-30 × ISI, ISR t60-210/glucose disposal t60-210 $\times$ ISI and ISR t0-210/glucose disposal t0-210 × ISI, respectively [22]. In addition, we calculated the ratio of late phase DI to early phase DI, based on earlier observations marking their relationship [23].

To improve comparison with previous studies, we also added a large number of classical indices to online supplemental Table 1 and supplemental Figure 1. All formulae are described below the online supplemental Table 1. All OGTT indices were derived from insulin and C-peptide concentrations in pmol/L and glucose concentrations in $\mathrm{mmol} / \mathrm{L}$, with the exception that insulin concentrations were converted to $\mu \mathrm{U} / \mathrm{mL}$ for the calculation of HOMA and ISI and, subsequently, DIs were both calculated with glucose in mg/dL. All AUCs were calculated according to the trapezoid method, and incremental AUCs were calculated by subtracting basal values from total calculated AUC values between given time points [24]. 
Table 1 Clinical characteristics of the NGT, IGT and/or IFG and T2D subgroups

\begin{tabular}{|c|c|c|c|c|c|c|}
\hline & NGT SA & NGT Cau & IFG/IGT SA & IFG/IGT Cau & T2D SA & $\mathrm{T} 2 \mathrm{D} \mathrm{Cau}$ \\
\hline$n$ & 22 & 34 & 12 & 12 & 23 & 18 \\
\hline Sex(male/female), $n \%$ (male) & $10 / 12(45.5)$ & $11 / 23(32.4)$ & $8 / 4(66.7)$ & $4 / 8(33.3)$ & $11 / 12(47.8)$ & $9 / 9(50.0)$ \\
\hline Age (years) & $39.6 \pm 11.6^{\top}$ & $38.9 \pm 9.4^{*}$ & $46.3 \pm 8.8$ & $44.5 \pm 11.4^{\ddagger}$ & $52.3 \pm 8.8^{* 8}$ & $63.2 \pm 7.6 * \uparrow$ \\
\hline Weight (kg) & $78.7 \pm 13.8$ & $81.1 \pm 15.7$ & $78.7 \pm 14.5$ & $94.1 \pm 30.4$ & $74.4 \pm 12.4$ & $90.5 \pm 15.0^{\bullet}$ \\
\hline Length $(\mathrm{cm})$ & $1.69 \pm 0.1$ & $1.75 \pm 0.1$ & $1.67 \pm 0.1$ & $1.75 \pm 0.1$ & $1.61 \pm 0.1^{*}$ & $1.76 \pm 0.1^{\oplus}$ \\
\hline $\operatorname{BMI}\left(\mathrm{kg} / \mathrm{m}^{2}\right)$ & $27.6 \pm 4.1$ & $26.3 \pm 4.1$ & $27.9 \pm 2.9$ & $30.4 \pm 8.5$ & $28.6 \pm 4.1$ & $29.3 \pm 4.9$ \\
\hline Waist $(\mathrm{cm})$ & $94 \pm 10$ & $91 \pm 15^{*}$ & $98 \pm 13$ & $105 \pm 20$ & $97 \pm 11$ & $105 \pm 14^{*}$ \\
\hline Hip (cm) & $105 \pm 5$ & $108 \pm 8$ & $103 \pm 6$ & $116 \pm 20$ & $104 \pm 9$ & $112 \pm 10$ \\
\hline $\mathrm{W} / \mathrm{H}$ & $0.90 \pm 0.07$ & $0.84 \pm 0.09^{*}$ & $0.95 \pm 0.10$ & $0.90 \pm 0.07$ & $0.93 \pm 0.08$ & $0.94 \pm 0.08 *$ \\
\hline RR systolic (mmHg) & $122.6 \pm 14.1$ & $123.2 \pm 12.5$ & $125.9 \pm 15.0$ & $129.6 \pm 20.2$ & $132.4 \pm 15.1$ & $135.6 \pm 13.0$ \\
\hline RR diastolic (mmHg) & $77.0 \pm 9.7$ & $76.3 \pm 9.1$ & $85.0 \pm 10.8$ & $79.4 \pm 10.3$ & $80.4 \pm 8.4$ & $83.4 \pm 12.2$ \\
\hline Smoking, $n \%^{\mathrm{D}}$ & $2(11.8)$ & $13(40.6)$ & (5) 55.6 & (5) 50.0 & $8(50.0)$ & $5(50.0)$ \\
\hline Antihypertensive, $n \%$ & $2(9.1)$ & $0^{*}$ & $2(16.7)$ & $3(25.0)$ & $9(39.1)$ & $9(50.0)^{*}$ \\
\hline Lipid treatment, $n \%$ & $3(13.6)$ & $1(2.9)^{*}$ & $3(25.0)$ & $0^{*}$ & $11(47.8)$ & $11(61.1)^{* \dagger}$ \\
\hline Macrovascular history, $n \%$ & 4.5 & 3.0 & 16.7 & 0 & 8.7 & $12.5^{*}$ \\
\hline Microvascular history, $n \%$ & & & & & $13.0^{\mathrm{B}}$ & $25.0^{\mathrm{B}}$ \\
\hline Metformin usage & & & & & $20(87 \%)$ & $17(94.4 \%)$ \\
\hline Age of diagnosis & & & & & $44.3 \pm 7.3^{\mathrm{A}, \mathrm{B}}$ & $56.1 \pm 7.2^{\mathrm{A}, \mathrm{B}}$ \\
\hline Period of having T2D & & & & & $9.9 \pm 7.3^{\mathrm{A}, \mathrm{C}}$ & $8.5 \pm 8.4^{\mathrm{A}, \mathrm{C}}$ \\
\hline Fasting glucose (mmol/L) & $5.3 \pm 0.4$ & $5.2 \pm 0.3^{\dagger *}$ & $6.0 \pm 0.5^{\bullet}$ & $6.0 \pm 0.6^{* *}$ & $7.2 \pm 1.1^{\S ॥}$ & $8.0 \pm 1.1^{* \dagger}$ \\
\hline $120 \mathrm{~min}$ glucose $(\mathrm{mmol} / \mathrm{L})$ & $5.4 \pm 1.1^{\oplus}$ & $5.5 \pm 1.1^{*}$ & $7.8 \pm 1.1^{\curvearrowleft}$ & $7.7 \pm 2.3^{* *}$ & $12.5 \pm 4.4^{\S ॥}$ & $14.1 \pm 3.6^{* \dagger}$ \\
\hline ISI & $5.0 \pm 3.9$ & $8.2 \pm 5.1^{*}$ & $3.2 \pm 1.8$ & $5.0 \pm 3.2$ & $2.9 \pm 1.6$ & $4.2 \pm 3.3^{*}$ \\
\hline ISR t0-210 & $1,647 \pm 852$ & $1,153 \pm 385$ & $1,873 \pm 862$ & $1,369 \pm 561$ & $1,645 \pm 513^{*}$ & $1,060 \pm 478$ \\
\hline ISR t0-30 & $297 \pm 122 * \uparrow$ & $208 \pm 82^{*}$ & $254 \pm 158^{\bullet}$ & $166 \pm 69$ & $116 \pm 64^{\S \|}$ & $124 \pm 75$ \\
\hline ISR t60-210 & $921 \pm 632$ & $595 \pm 296$ & $1,217 \pm 699$ & $900 \pm 524$ & $1,225 \pm 430^{*}$ & $744 \pm 360$ \\
\hline Glucose disposal t0-210 & $169 \pm 95^{\bullet}$ & $211 \pm 126^{\ddagger}$ & $394 \pm 138^{\circ}$ & $446 \pm 176^{*}$ & $830 \pm 438^{\S \|}$ & $1,035 \pm 445^{* \dagger}$ \\
\hline Glucose disposal t0-30 & $33 \pm 17$ & $35 \pm 14^{*}$ & $40 \pm 14$ & $44 \pm 27^{*}$ & $51 \pm 22^{\S}$ & $70 \pm 23^{\dagger}$ \\
\hline Glucose disposal t60-210 & $73 \pm 60^{\bullet}$ & $99 \pm 81^{*}$ & $239 \pm 107^{\curvearrowleft}$ & $281 \pm 162^{*}$ & $611 \pm 383^{\S \|}$ & $764 \pm 405^{* \dagger}$ \\
\hline
\end{tabular}

Data are mean $\pm \mathrm{SD}, n$ or $n(\%) . P$ values are from ANOVA, $P$ values between subgroups in post hoc Bonferroni analysis denoting statistical significance $(P<0.0125)$ are shown with symbols; * versus Cau NGT, ${ }^{\dagger}$ versus Cau IFG/IGT, ${ }^{\star}$ versus Cau T2D, ${ }^{\S}$ versus SA NGT, "versus SA IFG/IGT, “versus SA T2D. A newly identified individuals with T2D excluded, ${ }^{\mathrm{B}}$ significance, ${ }^{\mathrm{C}}$ non-significance with Student's $t$ test or $\chi^{2}$ test $P<0.05,{ }^{D}$ incomplete data, however, with a $>75 \%$ response rate

\section{Statistical analyses}

We performed family-based analyses with the SOLAR software package [25]. Comparison between ethnicities was performed with variance component analyses adjusted for a number of covariates within SOLAR. For the prediction of NGT, IFG/IGT or T2D stage (WHO OGTT subgroup) in both ethnicities, we used ordinal regression analyses with SPSS version 15.0 for Windows (SPSS Inc., Chicago, IL, USA), adjusted for family ties, using a variable grouping each family with their own distinct number in SPSS. Data are expressed as mean $\pm \mathrm{SD}$, unless otherwise indicated. ANOVA were used for differences within given WHO OGTT subgroups and performed with SPSS; for each WHO OGTT subgroup, three comparisons were performed with ANOVA (unless otherwise stated); with the other two WHO OGTT subgroups of same ethnicity and with the corresponding other ethnic WHO OGTT subgroup. Inverse or log transformations were used when normality, or equal variance assumptions were not met. $P$ value $<0.05$ was considered significant, unless otherwise stated.

\section{Results}

In 36 South Asian families, 15 out of 37 (41\%) apparently healthy first-degree relatives were classified as IFG $(n=4)$, IGT $(n=5)$, the combination of IFG and IGT $(n=3)$ or newly diagnosed T2D $(n=3)$. In 24 Caucasian families, $13(28 \%)$ out of 47 apparently healthy firstdegree relatives were classified as IFG $(n=3)$, IGT 


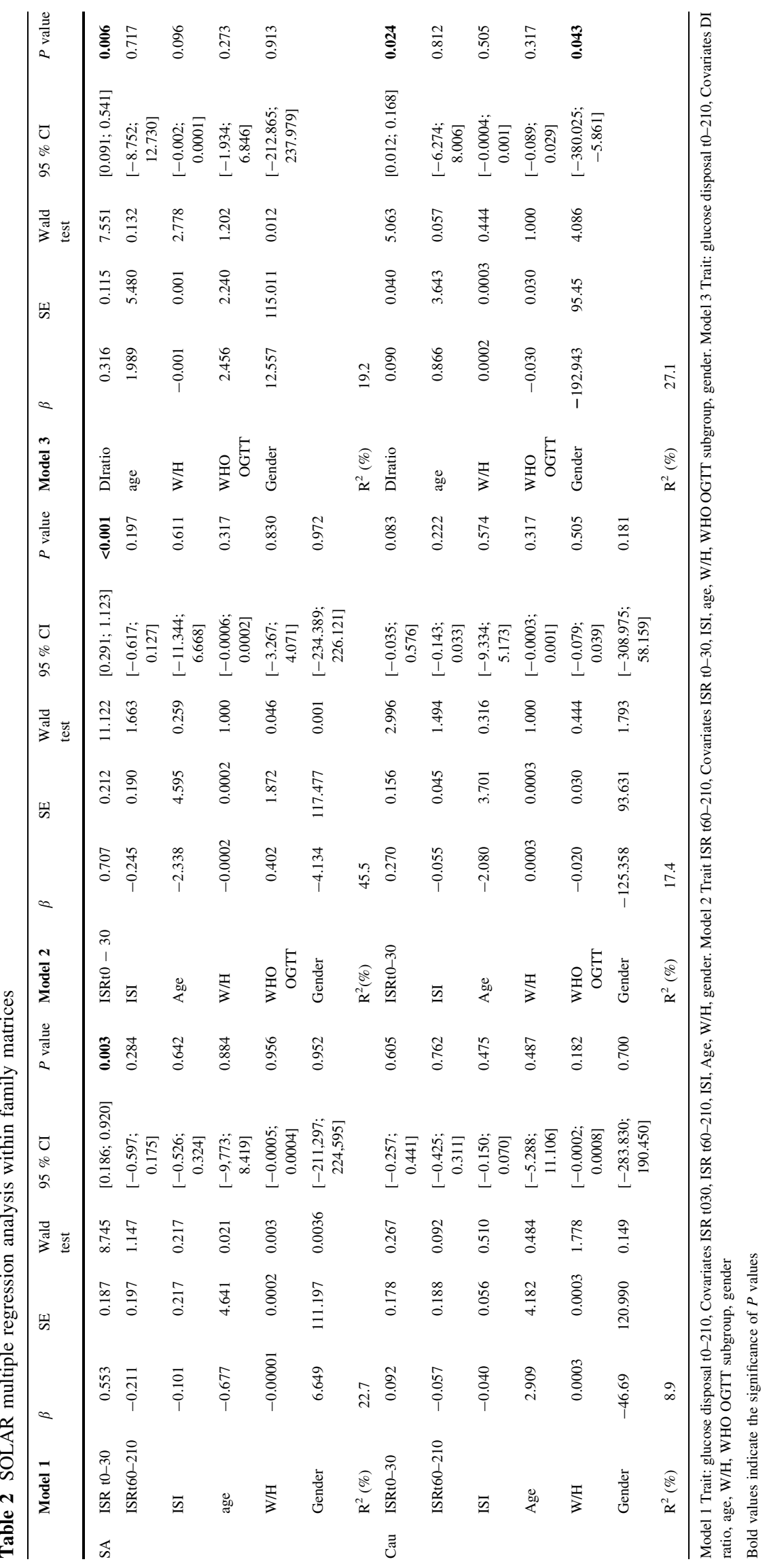


$(n=5)$, the combination of IFG and IGT $(n=4)$, while one was newly identified as T2D. For both ethnic groups, individuals with IFG and/or IGT were combined into one group of intermediate phenotypes and newly identified individuals with $\mathrm{T} 2 \mathrm{D}$ were included with the original T2D cases in the diabetes group. The general characteristics of the three groups according to ethnicity are shown in Table 1. Waist circumference and $\mathrm{W} / \mathrm{H}$ were lower in the groups with NGT compared with the other groups in both ethnicities. However, the relation of increasing $\mathrm{W} / \mathrm{H}$ with glucose intolerance appeared to be less clear in South Asians when compared to Caucasians Notably, the South Asians with T2D were on average 10 years younger than the Caucasians with T2D and they already had a substantial prevalence of macrovascular disorders. Results from the OGTT demonstrated the following results; in both ethnicities with increasing glucose intolerance, glucose disposal increased, while both ISR t0-30 min and ISI decreased. Both ISR t0-210 min and ISR t60-210 min increased from NGT toward IFG/IGT, but decreased from IFG/IGT toward T2D. In general, ISR derived parameters in South Asians were markedly higher compared with the Caucasians, while ISI was lower with an overall between ethnicity difference of $3.8 \pm 2.9$ versus $6.5 \pm 4.7$, respectively $(P<0.001)$.

Disposition indices, first/second phase beta-cell function

The unadjusted relationships between glucose disposal t0-210 min, ISR t0-30 min and ISR t60-210 min are shown in ternary plots (Supplementary Figure 1a, c). We determined the relationship between ISR t0-30 and ISR t60-210 with glucose disposal t0-210 using variance component analyses; after adjustment for ISI, age, W/H and gender the effect of early beta-cell function on glucose disposal t0-210 remained present in the South Asians, but disappeared in the Caucasian families, explaining the variance of glucose disposal t0-210 in our final model by 22.7 and $8.9 \%$ in South Asian and Caucasian families, respectively (Table 2, Model 1). We also explored the effect of ISR t0-30 and ISI on ISR t60-210. The unadjusted relationships between ISI, ISR t0-30 and ISR t60-210 are shown in the ternary plots of Supplementary Figure 1b, d. After adjustment for age, W/H,WHO OGTT subgroup and gender, ISR t0-30 in South Asians had an effect on ISR t60-210, but such effects were not observed in Caucasian families, explaining 45.5 and $17.4 \%$ of the variance of ISR t60-210 in our final model in South Asian and Caucasian families, respectively (Table 2, Model 2). We combined both ethnicities into an overall group and applied both Model 1 and 2 and tested for interaction between ethnicity and ISR t0-30 to glucose disposal

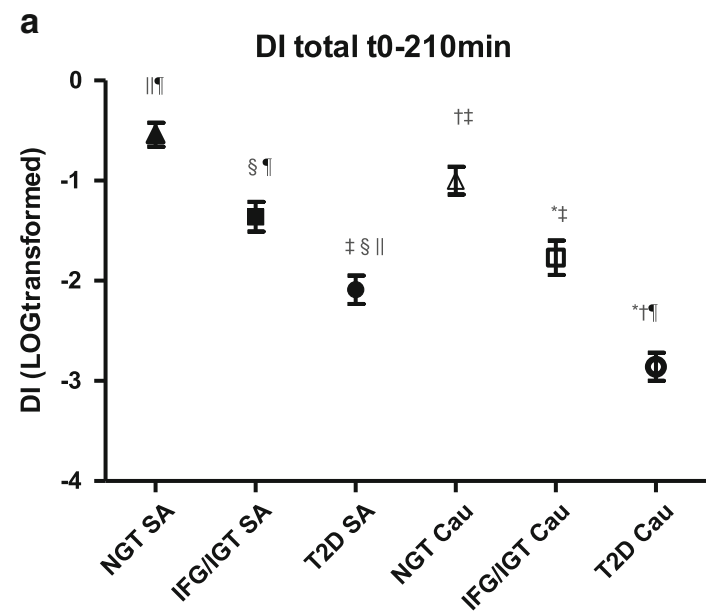

\section{b biphasic DI of SA and Cau subgroups}

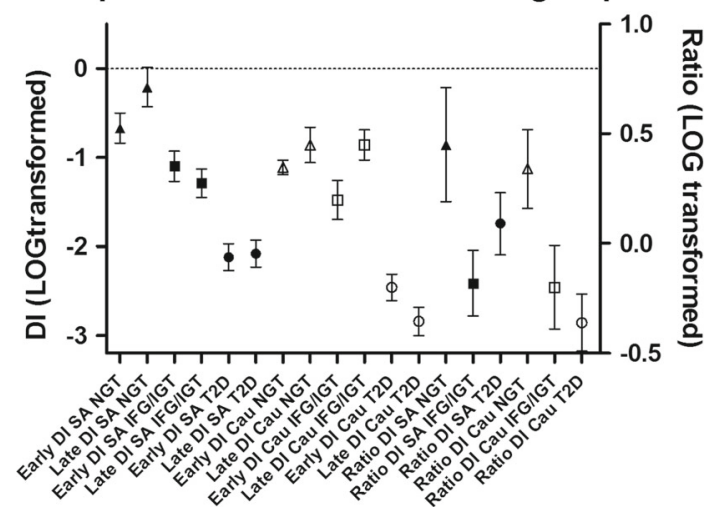

Fig. 1 a Overall DI (DI t0-210) for all WHO OGTT subgroups (mean \pm SEM) of both South Asian (closed) and Caucasian (open) families (triangle represents NGT, square IFG/IGT and circle T2D for both ethnicities). $P$ values between subgroups in post hoc Bonferroni analysis denoting statistical significance $(P<0.0125)$ are shown with symbols; *versus Cau NGT, "versus Cau IFG/IGT, ${ }^{\star}$ versus Cau T2D, ${ }^{\S}$ versus SA NGT, "versus SA IFG/IGT, "versus SA T2D. b Early DI (DI t0-30) and late DI (t60-210) on left $Y$ axis in mean $\pm \mathrm{SEM}$, and ratio of late phase/early phase DI (right $Y$ axis, mean \pm SEM) for NGT, IFG/IGT and T2D of both South Asian (closed) and Caucasian (open) families (triangle represents NGT, square IFG/IGT and circle T2D for both ethnicities). South Asians: In early DI, there was a significant difference between NGT versus T2D and IFG/IGT versus T2D $(P<0.0001)$. In late DI, there was a significant difference between NGT versus IFG/IGT, NGT versus T2D and IFG/IGT versus T2D $(P<0.0001)$. In DI ratio, no significant differences were found $(P=0.14)$. Caucasians: In both early and late DI, there was a significant difference between NGT versus T2D and IFG/IGT versus T2D (both $P<0.0001$, respectively). In DI ratio, there was a significant difference between NGT versus T2D $(P=0.016)$

t0-210 or ISR t60-210, respectively; only in Model 2, this interaction was significant $(\beta=0.341,[0.018 ; 0.664])$.

The overall DIs after logarithmic transformation of the three WHO OGTT subgroups according to ethnicity are shown in Fig. 1a. In both ethnicities, the overall DI decreased from the NGT to the IFG/IGT and further to the 


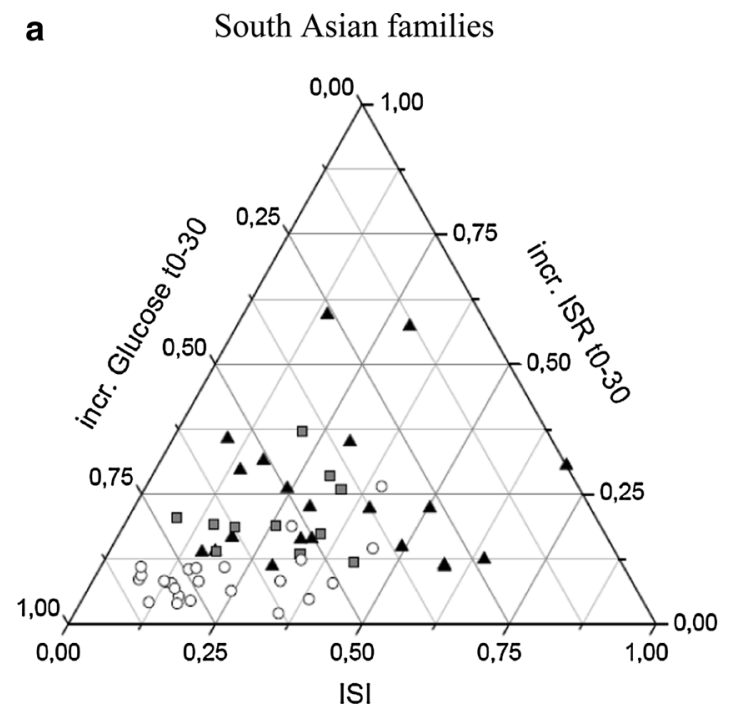

Fig. 2 a, b Ternary plot of relationship between insulin sensitivity (ISI), early phase beta-cell function (ISR t0-30) and glucose disposal (incr. glucose AUC t0-30) based on OGTT from South Asian (figures

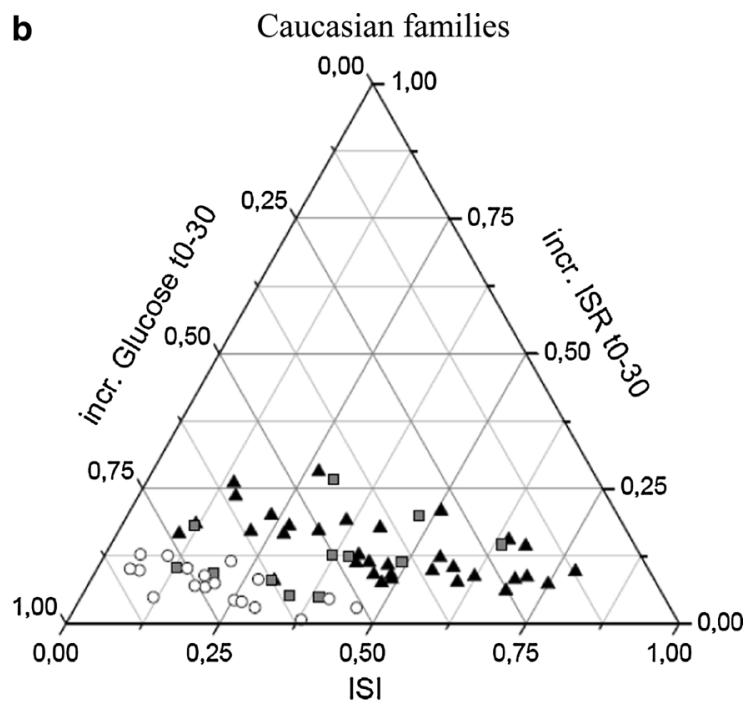

left) and Caucasian families (triangle represents NGT, square IFG/ IGT and circle T2D for both ethnicities)
Table 3 Ordinal and binary logistic regression analysis in both ethnicities predicting WHO OGTT subgroups adjusted for family ties
Bold values indicate the significance of $P$ values

\begin{tabular}{|c|c|c|c|c|c|}
\hline Independent & $\mathrm{B}$ & SE & Wald & $95 \% \mathrm{CI}$ & $P$ value \\
\hline \multicolumn{6}{|c|}{ Ordinal regression analysis with NGT, IFG/IGT and T2D as dependent variables } \\
\hline \multicolumn{6}{|l|}{ SA } \\
\hline ISR t0-30 & -0.020 & 0.009 & 5.246 & {$[-0.037 ;-0.002]$} & 0.022 \\
\hline ISI & -0.782 & 0.582 & 1.806 & {$[-1.922 ; 0.359]$} & 0.179 \\
\hline \multicolumn{6}{|l|}{$\mathrm{Cau}$} \\
\hline ISR t0-30 & -0.066 & 0.020 & 11.128 & {$[-0.105 ;-0.027]$} & 0.001 \\
\hline ISI & -0.603 & 0.256 & 5.556 & {$[-1.105 ;-0.101]$} & 0.018 \\
\hline \multicolumn{6}{|c|}{ Binary logistic regression analysis with $\mathrm{T} 2 \mathrm{D} / \mathrm{non} \mathrm{T} 2 \mathrm{D}$ as dependent variable } \\
\hline \multicolumn{6}{|l|}{ SA } \\
\hline ISR t0-30 & -0.029 & 0.009 & 11.350 & {$[-0.047 ;-0.011]$} & 0.001 \\
\hline ISI & -0.338 & 0.275 & 1.516 & {$[-0.877 ; 0.201]$} & 0.218 \\
\hline \multicolumn{6}{|l|}{$\mathrm{Cau}$} \\
\hline ISR t0-30 & -0.017 & 0.006 & 7.859 & {$[-0.029 ;-0.005]$} & 0.005 \\
\hline ISI & -0.319 & 0.124 & 6.630 & {$[-0.562 ;-0.076]$} & 0.01 \\
\hline
\end{tabular}

Adjusted for age, W/H, WHO OGTT subgroup and gender, DI ratio had an effect on glucose disposal t0-210 in both ethnicities, with the explained variances of glucose disposal t0-210 in our final model of 19.2 and $27.1 \%$ in South Asian and Caucasian families, respectively (Table 2, Model 3). However, gender also played a role in Caucasian families, but not in South Asian families.

Finally, to explore the differences in glucose handling in the WHO OGTT subgroups within the ethnicities, ternaries based on early DI are shown in Fig. 2a-b. (for total overview, three ternaries based on overall, early and late DI are shown in online Supplementary Fig. 2a-f). We used ordinal regression analyses to examine the nature of the components forming early and late DI. The ordinal analyses based on 
early DI parameters to predict WHO OGTT subgroups, adjusted for family ties, are given in Table 3 . In contrast to Caucasians, there was an exclusive role for early beta-cell function, and not ISI, in predicting glucose tolerance in the South Asian families. Even when including early glucose disposal (glucose disposal t0-30) as an additional covariate in the analysis, ISR t0-30 remained the single significant predictor. In the Caucasian families, both ISR t0-30 and ISI contributed significantly. For both ethnicities, similar ordinal analyses based on late DI parameters did not show significant effects with the exception of late glucose disposal t60-210 (data not shown). Figure $2 \mathrm{a}-\mathrm{b}$ suggest that the groups with T2D occupy a more distinct area toward the left corner of the ternaries, whereas the other two groups overlap more in the center. Therefore, we also performed logistic regression, adjusted for family ties, with the T2D groups versus the other relatives, the results can be found in Table 3; again, ISR t0-30 remained the most important discriminating variable in South Asians, even when glucose disposal t0-30 was included. For both ethnicities, similar binary logistic regression analyses based on late DI parameters demonstrated glucose disposal t60-210 as the most discriminating variable (data not shown).

\section{Discussion}

Across WHO OGTT subgroups from South Asian families, including the NGT group, we observed more insulin resistance, with more rapid decline of both early and late DI in NGT toward IFG/IGT, suggestive of early onset beta-cell failure. Across the WHO OGTT subgroups in Caucasian families, we observed a clear trend from normal insulin sensitivity to insulin resistance, while the DI decreased. Among the South Asians, the early insulin response explained at least partly the late insulin response as well as the overall glucose disposal. The ratio of the late over early DI decreased in both ethnicities from NTG to IFG/IGT, but waxed in the South Asian T2D and waned in the Caucasian T2D group, resulting in significant, but opposing effects in both ethnicities on the overall glucose disposal. The South Asians developed overt T2D at young age, while they still had a relatively high DI ratios. As a result of the lack of variance between the South Asian WHO OGTT subgroups in insulin sensitivity, only the early ISR predicted glucose tolerance state. Taken together, our findings confirm that changes in beta-cell dynamics play a prominent role in the development of T2D in South Asians. In Caucasians, more gradual processes of increasing resistance to insulin and decreasing overall insulin secretion seem to take place.

Our data confirm that-without adjustment for insulin sensitivity—South Asian individuals wrongly seem to have enough beta-cell capacity (Supplementary Table 1) with an above-average ability to secrete insulin [15]. Unfortunately, this compensatory beta-cell function is insufficient, leading to very early onset of T2D, as shown by the young age of manifest T2D in South Asians. These observations underline the important role of changes in beta-cell function, which have been reported to be the main contributor to abnormal glucose tolerance among a wide range of ethnicities, and are in line with increasing genetic evidence for beta-cell defects as an important predisposing factor for T2D [16, 17]. Hypersecretion of insulin may reflect betacell responses to different signals or a combination of an increased potentiating effect of glucose on beta-cells, longlasting adaptation to severe insulin resistance and/or problems with the processing of insulin.

Clamp studies have demonstrated decreased insulin sensitivity among healthy South Asians when compared to other healthy controls [26-29]. We also found a decreased insulin sensitivity in the South Asians compared with the Caucasians. In contrast to the Caucasians, the degree of insulin sensitivity did not change between the three South Asian WHO OGTT subgroups. This very strong familial aggregation of insulin resistance suggests a strong contribution of environmental factors. However, we cannot infer from our data whether lifestyle, type of food, microbiome or other factors are involved. Among our families with high risk of T2D, the South Asians had much earlier onset of signs and symptoms of T2D compared with Caucasians. Notably, the burden from macrovascular disease was larger in our South Asian families, even in relatives who did not have T2D. This suggests that the severe insulin resistance of the South Asians contributes strongly to atherogenesis.

In addition to a demanding insulin resistant environment, failing beta-cell capacity is a major susceptibility factor to T2D in South Asian families, as was supported by recent genome wide association studies (GWAS). These studies show greater effects of SNP variants in beta-cell-related genes in South Asians than in other populations [30].

The strength of the present study is that it was a familybased approach and analysis of two ethnic groups, among various stages of glucose tolerance. Moreover, beta-cell and insulin sensitivity indices were based on multiple sampled prolonged OGTT's. The relatively small numbers within the WHO OGTT subgroups of the families are a potential weakness, but a characteristic of both extensive phenotyping and family analyses is that it can be performed in relatively small populations. In line, we were able to observe beta-cell function alterations in a very consistent way.

\section{Conclusion}

Based on extended OGTT measurements, we found that insulin sensitivity is already lower in South Asian than in 
Caucasian people with NGT. Insulin resistance in the South Asians does not change much during progression of glucose intolerance, and beta-cell dysfunction might play a dominant role in the early development of T2D among South Asian families in the Netherlands.

Acknowledgments We thank Dr. Hovorka for kindly providing us with the ISEC program.

Conflict of interest Sjaam Jainandunsing, Behiye Özcan, Trinet Rietveld., Joram N. I. van Miert, Aaron J. Isaacs, Janneke G. Langendonk, Felix W. M. de Rooij. and Eric J. G. Sijbrands declare that they have no conflict of interest.

Human and Animal Rights disclosure All procedures followed were in accordance with the ethical standards of the responsible committee on human experimentation (institutional and national) and with the Helsinki Declaration of 1975, as revised in 2008.

Informed consent disclosure Informed consent was obtained from all patients for being included in the study.

Open Access This article is distributed under the terms of the Creative Commons Attribution License which permits any use, distribution, and reproduction in any medium, provided the original author(s) and the source are credited.

\section{References}

1. Middelkoop BJ et al (1999) Diabetes mellitus among South Asian inhabitants of The Hague: high prevalence and an age-specific socioeconomic gradient. Int J Epidemiol 28(6):1119-1123

2. Bindraban NR et al (2008) Prevalence of diabetes mellitus and the performance of a risk score among Hindustani Surinamese, African Surinamese and ethnic Dutch: a cross-sectional population-based study. BMC Public Health 8:271

3. Shaw PKC et al (2006) South-Asian type 2 diabetic patients have higher incidence and faster progression of renal disease compared with Dutch-European diabetic patients. Diabetes Care 29(6): 1383-1385

4. Bindraban NR et al (2008) A new tool, a better tool? Prevalence and performance of the International Diabetes Federation and the National Cholesterol Education Program criteria for metabolic syndrome in different ethnic groups. Eur J Epidemiol 23(1):37-44

5. Schreuder YJ et al (2011) Ethnic differences in maternal total cholesterol and triglyceride levels during pregnancy: the contribution of demographics, behavioural factors and clinical characteristics. Eur J Clin Nutr 65(5):580-589

6. Troe EJ et al (2007) Explaining differences in birthweight between ethnic populations. Generation R Study. Bjog 114(12):1557-1565

7. van Steijn L et al (2009) Neonatal anthropometry: thin-fat phenotype in fourth to fifth generation South Asian neonates in Surinam. Int J Obes (Lond) 33(11):1326-1329

8. Shaw PKC et al (2007) Central obesity is an independent risk factor for albuminuria in nondiabetic South Asian subjects. Diabetes Care 30(7):1840-1844

9. Agyemang $\mathrm{C}$ et al (2010) Educational inequalities in metabolic syndrome vary by ethnic group: Evidence from the SUNSET study. Int J Cardiol 141(3):266-274

10. Middelkoop BJ, van der Wal G (2004) Culture-specific diabetes care for Surinam South Asians with a low socio-economic position: who benefits? Patient Educ Couns 53(3):353-358
11. Denktas S et al (2009) Ethnic background and differences in health care use: a national cross-sectional study of native Dutch and immigrant elderly in the Netherlands. Int J Equity Health 8:35

12. Wulan SN, Westerterp KR, Plasqui G (2010) Ethnic differences in body composition and the associated metabolic profile: a comparative study between Asians and Caucasians. Maturitas 65(4):315-319

13. Lear SA et al (2009) Ethnic variation in fat and lean body mass and the association with insulin resistance. J Clin Endocrinol Metab 94(12):4696-4702

14. Abate $\mathrm{N}$ et al (2004) Adipose tissue metabolites and insulin resistance in nondiabetic Asian Indian men. J Clin Endocrinol Metab 89(6):2750-2755

15. McKeigue PM, Shah B, Marmot MG (1991) Relation of central obesity and insulin resistance with high diabetes prevalence and cardiovascular risk in South Asians. Lancet 337(8738):382-386

16. Jensen CC et al (2002) Beta-cell function is a major contributor to oral glucose tolerance in high-risk relatives of four ethnic groups in the U.S. Diabetes 51(7):2170-2178

17. McCarthy MI (2010) Genomics, type 2 diabetes, and obesity. N Engl J Med 363(24):2339-2350

18. Alberti KG, Zimmet PZ (1998) Definition, diagnosis and classification of diabetes mellitus and its complications. Part 1: diagnosis and classification of diabetes mellitus provisional report of a WHO consultation. Diabet Med 15(7):539-553

19. Hovorka R, Soons PA, Young MA (1996) ISEC: a program to calculate insulin secretion. Comput Methods Programs Biomed 50(3):253-264

20. Matsuda M, DeFronzo RA (1999) Insulin sensitivity indices obtained from oral glucose tolerance testing: comparison with the euglycemic insulin clamp. Diabetes Care 22(9):1462-1470

21. Matthews DR et al (1985) Homeostasis model assessment: insulin resistance and beta-cell function from fasting plasma glucose and insulin concentrations in man. Diabetologia 28(7):412-419

22. Abdul-Ghani MA et al (2008) The relationship between fasting hyperglycemia and insulin secretion in subjects with normal or impaired glucose tolerance. Am J Physiol Endocrinol Metab 295(2):E401-E406

23. Gerich JE (2002) Is reduced first-phase insulin release the earliest detectable abnormality in individuals destined to develop type 2 diabetes? Diabetes 51(Suppl 1):S117-S121

24. Purves RD (1992) Optimum numerical integration methods for estimation of area-under-the-curve (AUC) and area-under-themoment-curve (AUMC). J Pharmacokinet Biopharm 20(3):211-226

25. Almasy L, Blangero J (1998) Multipoint quantitative-trait linkage analysis in general pedigrees. Am J Hum Genet 62(5):1198-1211

26. Raji A et al (2001) Body fat distribution and insulin resistance in healthy Asian Indians and Caucasians. J Clin Endocrinol Metab 86(11):5366-5371

27. Chandalia M et al (1999) Relationship between generalized and upper body obesity to insulin resistance in Asian Indian men. J Clin Endocrinol Metab 84(7):2329-2335

28. Chandalia $M$ et al (2007) Insulin resistance and body fat distribution in South Asian men compared to Caucasian men. PLoS One 2(8):e812

29. Banerji MA et al (1999) Body composition, visceral fat, leptin, and insulin resistance in Asian Indian men. J Clin Endocrinol Metab 84(1):137-144

30. Kooner JS et al (2011) Genome-wide association study in individuals of South Asian ancestry identifies six new type 2 diabetes susceptibility loci. Nat Genet 43(10):984-989

31. Gauderman WJ (2002) Sample size requirements for matched case-control studies of gene-environment interaction. Stat Med 21(1):35-50 